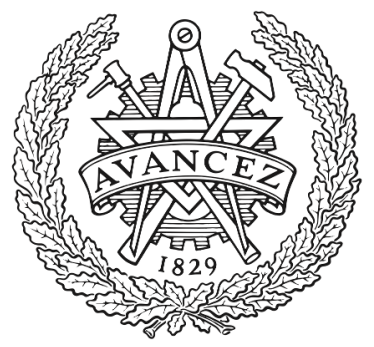

CHALMERS

UNIVERSITY OF TECHNOLOGY

\title{
Solvent Effects for Methanol Electrooxidation on Gold
}

Downloaded from: https://research.chalmers.se, 2023-04-26 09:08 UTC

Citation for the original published paper (version of record):

Valter, M., Wickman, B., Hellman, A. (2021). Solvent Effects for Methanol Electrooxidation on

Gold. Journal of Physical Chemistry C, 125(2): 1355-1360.

http://dx.doi.org/10.1021/acs.jpcc.0c08923

N.B. When citing this work, cite the original published paper. 


\title{
Solvent Effects for Methanol Electrooxidation on Gold
}

\author{
Published as part of The Journal of Physical Chemistry virtual special issue "Emily A. Carter Festschrift". \\ Mikael Valter, Björn Wickman, and Anders Hellman*
}

Cite This: J. Phys. Chem. C 2021, 125, 1355-1360

Read Online

\section{ACCESS | Lill Metrics \& More | 喟 Article Recommendations | S1 Supporting Information}

ABSTRACT: A detailed understanding of the methanol electrooxidation reaction mechanism is important for the further development of methanol fuel cells. By modeling the reaction on $\mathrm{Au}(111)$ using density functional calculations, we investigate the impact of solvent models, focusing on the potential-determining step and the theoretical limiting potential. Both implicit solvent effects, in the form of VASPsol, and explicit solvation by water molecules are investigated. The use of explicit water molecules changes the energetics of the reaction intermediates, and it

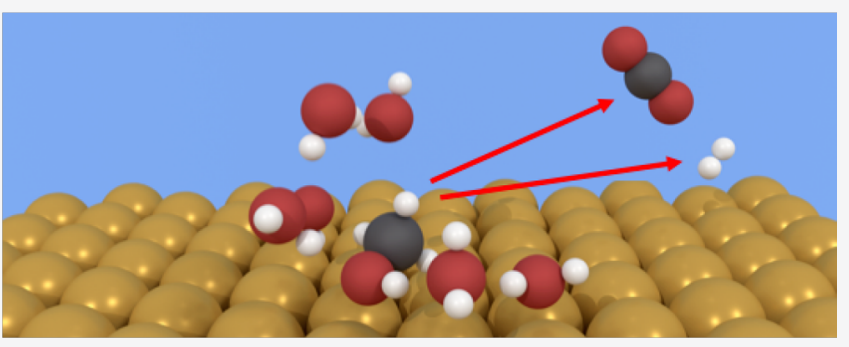
requires the addition of six water molecules to reach converged results. An important observation is that the configuration space of the explicit water molecules needs to be treated carefully. Upon comparison of the most simple vacuum model with a more advanced combined solvent model, it is clear that there are some pronounced differences; for instance, both implicit solvent effects and explicit solvation stabilize $\mathrm{HCOOH}$ and destabilize $\mathrm{CO}_{2}$. There are, however, qualitative agreements between the models; for instance, the first deprotonation step of methanol is found to be the potential-determining step, although the more accurate model put forth aldehyde and formate formation as possible competitive steps. The results are experimentally validated by using cyclic voltammetry.

\section{INTRODUCTION}

Over the years, direct methanol fuel cells (DMFCs) have gained increased interest, both scientifically and industrially, as an alternative to hydrogen-driven fuel cells. ${ }^{1-3}$ Being a liquid under ambient conditions, methanol has a higher energy content per volume, is more practical to store, and can utilize existing infrastructure for its distribution as compared to hydrogen. ${ }^{4}$ DMFCs are a key technology in an envisioned methanol economy. ${ }^{5}$

In the heart of DMFCs is the methanol oxidation reaction (MOR). MOR is a six-electron-transfer process involving water, which occurs at a potential of $0.02 \mathrm{~V}_{\text {vs }} \mathrm{RHE}^{6}$ at the anode side of a DMFC, and can be described as

$$
\mathrm{CH}_{3} \mathrm{OH}+\mathrm{H}_{2} \mathrm{O} \rightarrow \mathrm{CO}_{2}+6 \mathrm{H}^{+}+6 \mathrm{e}^{-}
$$

Owing to the many reaction intermediates involved, there are some restrictions as to the actual choice of catalyst. For instance, the anode should not bind the reaction intermediates too strongly but, at the same time, allow for the activation of water. ${ }^{7}$ Density functional calculations have been used to study methanol oxidation on different transition metals, including gold. ${ }^{8-12}$ Of the pure elements, Pt stands out as the best candidate $^{8,9}$ while bifunctional catalysts, based on $\mathrm{Pt}-\mathrm{Cu}$ and $\mathrm{Pt}-\mathrm{Ru}$ alloys, are suggested to be even better for MOR. ${ }^{10,11}$ The nobility of Au not only makes it an active electrocatalyst because of its resistance to surface oxidation but also implies that adsorption is weak. However, as a model catalyst, Au still plays an important role.

Although previous studies have been fruitful in revealing details of the MOR and suggest new catalyst candidates, there are some concerns on the modeling details of solvent effects, which, at least as a first approximation, are typically neglected. ${ }^{8-11}$ Recently, solvent effects have been included, either implicitly or explicitly, to better describe the electrolyte environment. For instance, solvent effects in methanol electrooxidation on $\mathrm{Pt}$, modeled implicitly, were important because of the different hydrophilic behavior of the reaction intermediates. ${ }^{7}$ However, findings like this raise the question of whether even more advanced solvent models are needed. In the case of electrooxidation of formic acid, which is an intermediate in MOR, a series of studies ${ }^{13-15}$ show that the inclusion of solvent effect is critical and can result in, for example, a change of the dominant reaction pathway. In a recent study Heenen et al. ${ }^{16}$ showed that the adsorption energy of some prototypical C1 and C2 species are different if evaluated by using implicit solvent models, which do not

Received: September 30, 2020

Revised: December 7, 2020

Published: January 8, 2021 
account for directional interactions, or by using ab initio molecular dynamics, which use explicit water molecules in the simulation. Explicit models such as microsolvation by single or few water molecules have been investigated, capturing the contribution of coadsorbed water for $* \mathrm{OH}$ and $* \mathrm{OOH}$ groups on platinum nanoparticles. ${ }^{17}$ In the case of methanol oxidation on $\mathrm{Ru}, \mathrm{Pd}$, and $\mathrm{Pt}$, Garcia et al. argue that the use of two water molecules is sufficient to get converged results. It should be noted that the reaction intermediates in methanol oxidation only consist of $\mathrm{C} 1$ species, and for instance, Ludwig et al. ${ }^{18}$ found that for larger $\mathrm{C} 2$ species, for example, OCCO and $\mathrm{OCCHO}$, there is a noticeable difference between using five or ten explicit water molecules. As the use of explicit water molecules drastically increases the complexity, there are efforts to include solvent effects via corrections based on linear regression that can account for implicit and explicit solvent effects. ${ }^{19}$ However, despite recent developments, there is still no clear guidance on how to treat the electrolyte environment, implicitly, explicitly, or a combination of the two, particularly on a reaction that involves both hydrophilic and hydrophobic intermediates.

In this work, we investigate the importance of solvent effects for the thermodynamics of the methanol electrooxidation mechanism on $\mathrm{Au}(111)$ with DFT. This is achieved by using an implicit model, VASPsol, and explicit water molecules. We consider the reaction path for methanol electrooxidation to $\mathrm{CO}_{2}$ on $\mathrm{Au}(111)$ reported by Ferrin and Mavrikakis:

$$
\begin{aligned}
& \mathrm{CH}_{3} \mathrm{OH} \rightarrow \mathrm{CH}_{2} \mathrm{OH} \rightarrow \mathrm{CH}_{2} \mathrm{O} \rightarrow \mathrm{CHO} \rightarrow \mathrm{HCOOH} \\
& \quad \rightarrow \mathrm{CHOO} \rightarrow \mathrm{CO}_{2}
\end{aligned}
$$

where the first deprotonation is potential-determining. ${ }^{9}$ In addition to this, we investigate whether the route could go through the other possible intermediate, $\mathrm{CH}_{3} \mathrm{O}$, when solvent effects are taken into account.

\section{METHOD}

Density functional calculations were performed by using VASP, ${ }^{20-22}$ with the optB86b-vdW exchange-correlation functional. ${ }^{23-25}$ Inclusion of van der Waals is important for the description of methanol adsorption on gold, and this functional was chosen as it gives accurate methanol adsorption energy. ${ }^{26}$ The projector augmented wave method ${ }^{27}$ was used to model the interaction between the valence electrons and the core. Solvent effects were taken into account by using up to six explicit water molecules, and the structures were calculated with and without implicit solvent as implemented in VASPsol. $^{28,29}$ The Kohn-Sham orbitals were represented by using a plane-wave basis set with $450 \mathrm{eV}$ as cutoff energy, and a Gaussian smearing of $0.05 \mathrm{eV}$ was applied to the Fermi level discontinuity.

The $\mathrm{Au}(111)$ surface was modeled as a four-layer $\mathrm{p}(3 \times 4)$ orthogonal supercell. To sample the configuration space for adsorbates solvated by several water molecules, ab initio molecular dynamics (AIMD) was performed. The model for this was the $\mathrm{Au}(111)$ surface with three water layers and a fixed, outer layer of water molecules with zero dipoles keeping the volume of the unit cell fixed. The periodic slabs and fixed water layers were separated by at least $10 \AA$. In the AIMD simulations, only the gamma point was used to sample the reciprocal space. A Nosé-Hoover thermostat maintained the temperature at $300 \mathrm{~K}$. Hydrogen was tritiated (i.e., set to $3 \times$ the mass of hydrogen) to allow a time step of 1 fs. The duration was $10 \mathrm{ps}$, starting from a thermalized configuration from a prerun of 2 ps, and the first 1 ps was not used in the analysis. Configurations separated by 1 ps were used for the adsorption calculations, as shown in Figure 1. In total, ten

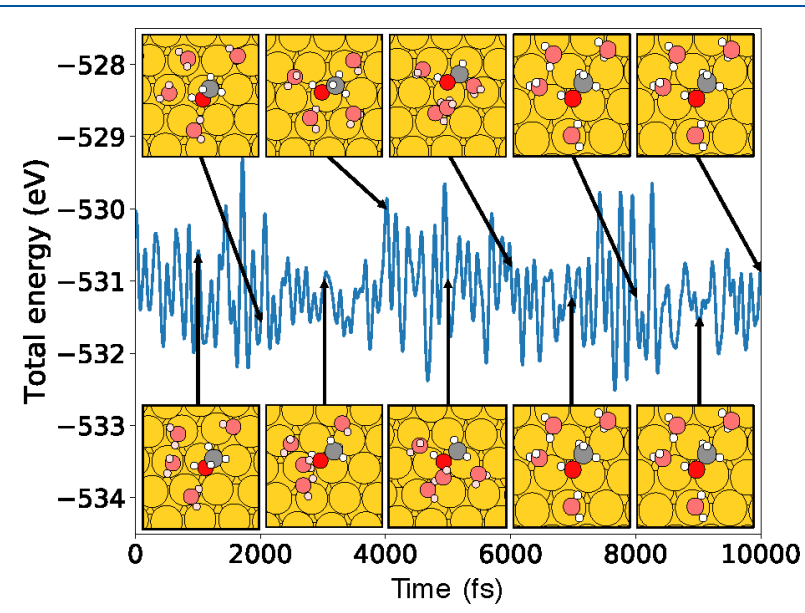

Figure 1. Example of the sampling process. From the AIMD simulation, images are selected 1 ps apart to be used in the adsorption calculation. The three water layers are removed except the $0-6$ ( 4 in this figure) closest water molecules to the adsorbate.

configurations were taken from the trajectory as starting configurations for finding global minima. From these configurations, we generated structures with a defined amount of water surrounding the adsorbate of interest as well as without adsorbate as a reference. The choice of which water to keep in the simulation cell was made by using the geometric distance between any atom in the adsorbate and any atom in the water molecule. We exclude configurations that form longrange $2 \mathrm{D}$ water networks in the simulation cell as this adds superstitious stabilization in the use of explicit water (see the Supporting Information).

The adsorbate calculations were performed with $(6,5,1) k$ points in a Monkhorst-Pack grid. ${ }^{30}$ The periodic surface slabs, including intermediates and water when applicable, were separated by a vacuum of $20 \AA$. The gas-phase species were computed in a $20 \times 20 \times 20 \AA^{3}$ cell by using only the gamma point. The quasi-Newton method was used for structural relaxations with a total residual force of $0.05 \mathrm{eV} / \AA$ as the convergence criterion. The most stable minimum for each intermediate was used in the analysis. Single-point calculations without VASPsol, which we refer to as "vacuum" below, were performed on the relaxed structures.

Vibrational modes were calculated by diagonalization of the Hessian matrix, where the force derivatives were computed by using the central difference approximation with a displacement of $0.015 \AA$. Calculations were performed for gas-phase molecules of water, methanol, hydrogen, and carbon dioxide as well as the most stable adsorbate configurations with no explicit water molecules (with and without implicit solvent). Other adsorbate configurations were assumed to have the same vibrational modes.

The Gibbs free energy was calculated at $298 \mathrm{~K}$, at the corresponding vapor pressure for water $(3.165 \mathrm{kPa})$, at $0.001 \mathrm{~atm}$ for carbon dioxide, and at $1 \mathrm{~atm}$ hydrogen and methanol, treated as ideal gases. Adsorbates were treated in the harmonic approximation with only vibrational degrees of freedom and a cutoff frequency of $50 \mathrm{~cm}^{-1}$. 


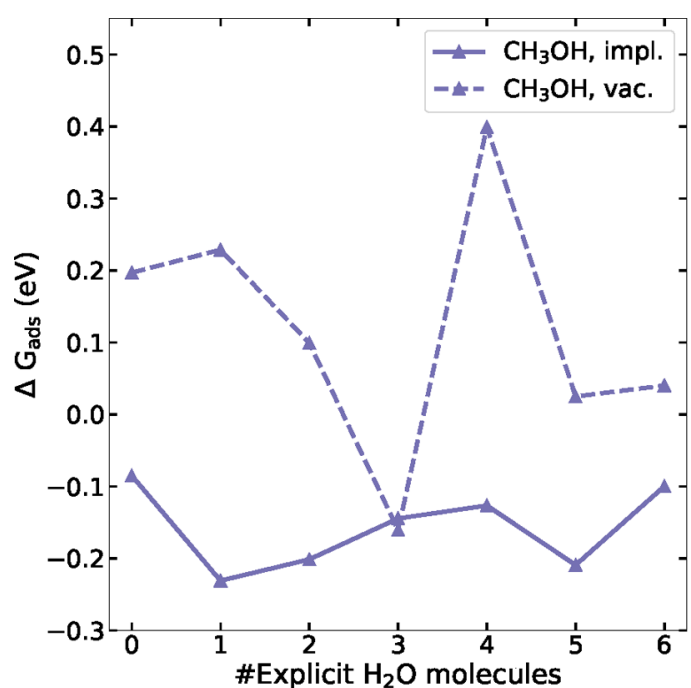

(a) Adsorption energy.

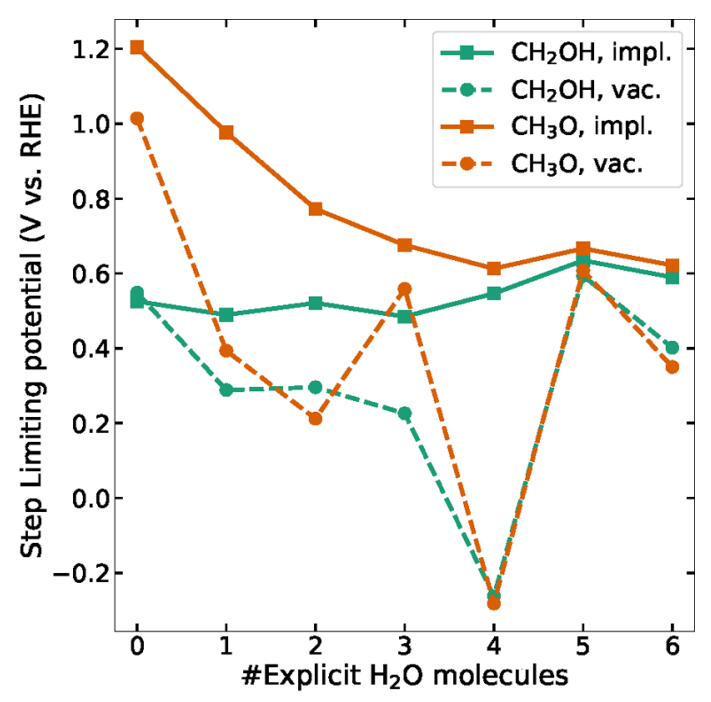

(b) Limiting potential of first deprotonation.

Figure 2. Solvent effects in (a) the Gibbs free energy of methanol adsorption and (b) the equilibrium potential for the first deprotonation step. Solid lines are with implicit solvent and dashed lines without implicit solvent. Energy is given in eV.

Electrochemical reactions were modeled by using the computational hydrogen electrode (CHE) by Rossmeisl and Nørskov, which assumes coupled electron-proton transfer. ${ }^{31}$ Here, zero potential is defined by using the reversible hydrogen electrode (RHE); that is, the free energy of hydrogen molecules (at 1 atm pressure) equals the energy of protons and electrons at all values of $\mathrm{pH}$. Any change in the potential $U_{\mathrm{RHE}}$ results in a shift of the free energy of the electrons with $\Delta G=-e U_{\mathrm{RHE}}$, where $e$ is the elementary charge. For example, we calculate the reaction energy of

$$
\mathrm{CH}_{3} \mathrm{OH} \rightarrow \mathrm{CH}_{2} \mathrm{OH}+\mathrm{H}^{+}+\mathrm{e}^{-}
$$

by calculating

$$
\mathrm{CH}_{3} \mathrm{OH} \rightarrow \mathrm{CH}_{2} \mathrm{OH}+\frac{1}{2} \mathrm{H}_{2}
$$

and adding $-e U_{\mathrm{RHE}}$.

It has traditionally been very challenging to calculate electrochemical barriers. One reason for this is that standard periodic DFT operates with a constant charge while an electrochemical process requires a variable charge. ${ }^{32}$ While there has been much development in how to calculate electrochemical barriers, ${ }^{32-35}$ we have decided to restrict ourselves to thermodynamics in this study.

Cyclic voltammetry was performed on a polycrystalline gold wire in a solution of $0.1 \mathrm{M} \mathrm{HClO}_{4}$ (Sigma-Aldrich, 70\%, ACS reagent grade, diluted with Milli-Q water) and $0.1 \mathrm{M} \mathrm{KOH}$ (Sigma-Aldrich, $\geq 85 \%$ purity, dissolved in Milli-Q water) and 0.5 M methanol (Sigma-Aldrich, 99.5\% purity). An $\mathrm{Ag} / \mathrm{AgCl}$ reference electrode (B3420+) from SI Analytics was used, and a graphite rod (Sigma-Aldrich, $99.995 \%$ purity) was used as counter electrode. The scan rate was $100 \mathrm{mV} / \mathrm{s}$. Nitrogen was bubbled to purge atmospheric oxygen $20 \mathrm{~min}$ before and during the experiments. The experiments were conducted with an SP-300 potentiostat from BioLogic Instruments.

\section{RESULTS AND DISCUSSION}

The adsorption Gibbs free energy of methanol was calculated by using 0-6 explicit water molecules and with and without the use of an implicit solvent model (Figure 2a). The reference is optimized water structures at the surface, only taking total energies into account. It can be seen that with implicit solvent the adsorption energy is not heavily dependent on the number of water molecules. However, the vacuum-calculated adsorption energy requires several water molecules to converge. It can be pointed out that the data point for four $\mathrm{H}_{2} \mathrm{O}$ is an outlier, such that the implicit-solvent relaxed structure is relatively unstable in a vacuum; a vacuum calculation on the second most stable implicit-solvent relaxed structure with four $\mathrm{H}_{2} \mathrm{O}$ gives $\Delta G_{\text {ads }}=0.02 \mathrm{eV}$, which would suggest a convergence for 3-6 water molecules. The overall trend is that the results with and without implicit solvent approaches each other as more and more water molecules are added.

The first deprotonation step of adsorbed methanol occurs by removing hydrogen from the carbon or oxygen in methanol. On $\mathrm{Au}(111)$, it was reported that preferably carbon is deprotonated and that this step is the potential-determining step. ${ }^{9}$ Thus, we are interested in solvent effects on the selectivity as well as the size of the step. The limiting potential of formation for $\mathrm{CH}_{2} \mathrm{OH}$ and $\mathrm{CH}_{3} \mathrm{O}$ (equal to free energy relative to adsorbed methanol divided by one electron) is shown as a function of implicit and explicit effects in Figure $2 \mathrm{~b}$. With implicit solvent, it is clear that $\mathrm{CH}_{3} \mathrm{O}$ is strongly affected by the inclusion of explicit water molecules, as the limiting potential changes from $1.20 \mathrm{~V}$ to 0.97 and $0.77 \mathrm{~V}$ for the first two water molecules, respectively, while the change is minor when adding more water molecules $\left(0.62 \mathrm{~V}\right.$ for six $\left.\mathrm{H}_{2} \mathrm{O}\right)$. In contrast, the change in limiting potential for the formation of $\mathrm{CH}_{2} \mathrm{OH}$ is less dependent on explicit water molecules $(0.55 \pm$ $0.08 \mathrm{~V}$ with implicit solvent). The vacuum calculations follow the same trends qualitatively, but the convergence is much slower with respect to explicit water molecules; the oscillatory behavior remains after taking the outlying data point for $\mathrm{CH}_{3} \mathrm{OH}$ with four $\mathrm{H}_{2} \mathrm{O}$ mentioned above into account. Furthermore, a more apparent trend is that the difference in limiting potential more or less disappears. The reason behind the larger stabilization of $\mathrm{CH}_{3} \mathrm{O}$ as compared to $\mathrm{CH}_{2} \mathrm{OH}$ is that $\mathrm{CH}_{3} \mathrm{O}$ typically forms hydrogen bonds from its oxygen to 


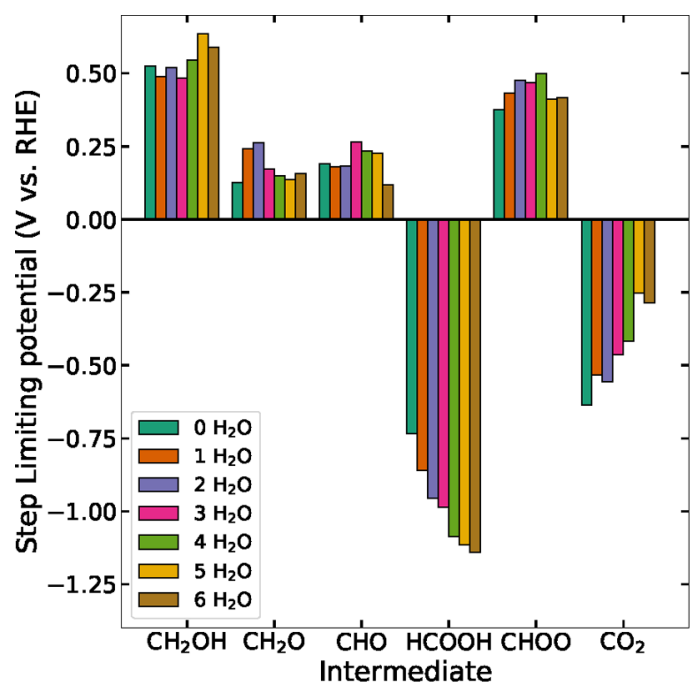

(a) Implicit solvent.

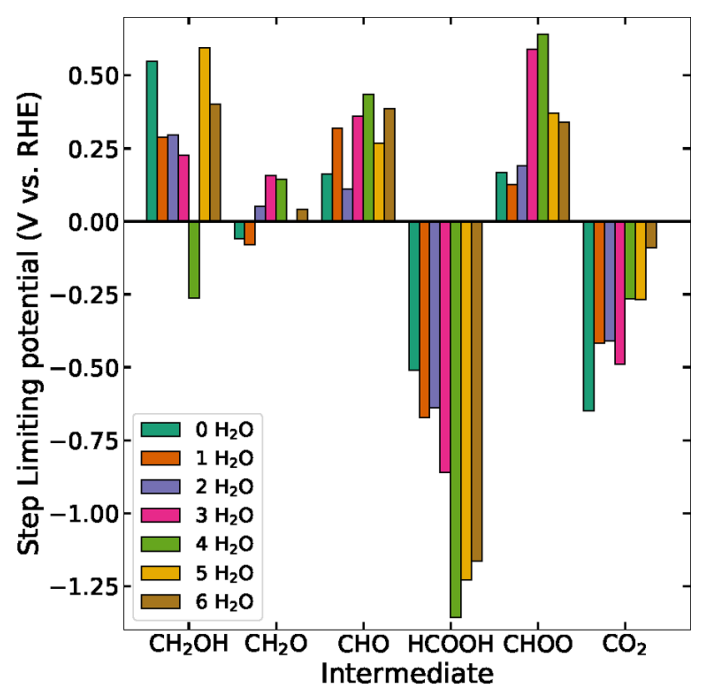

(b) Vacuum.

Figure 3. Equilibrium potential of formation of the methanol intermediates (a) with and (b) without implicit solvent. Energy is given in eV.

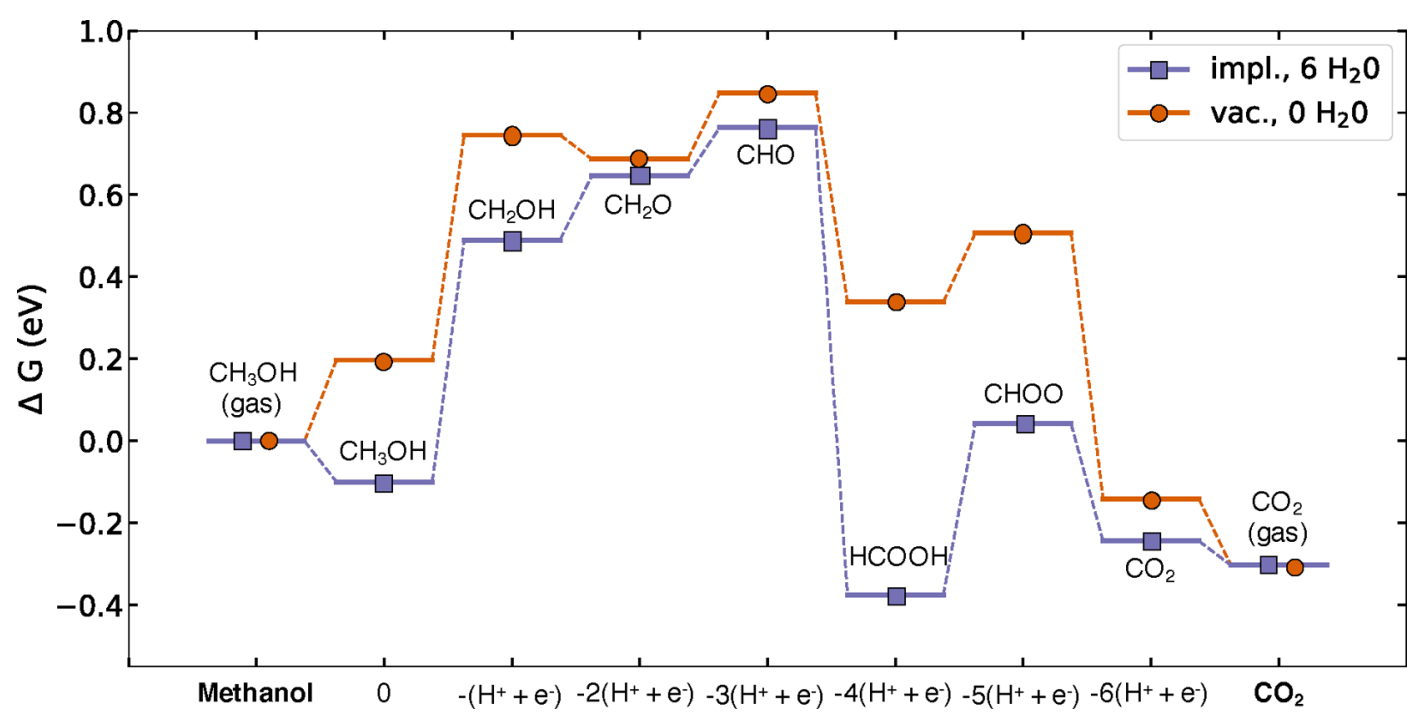

Figure 4. Energy landscape for methanol electrooxidation. The implicit model with six explicit water molecules (blue squares) is compared to calculation without implicit solvent (red circles). Energy is given in $\mathrm{eV}$.

two water molecules, while the hydroxy group in $\mathrm{CH}_{2} \mathrm{OH}$ only binds to one. It can be noted that $\mathrm{CH}_{3} \mathrm{O}$ must bind atop for this to occur, while the most stable site in the absence of explicit water molecules is a hollow site. We could, at this point, note that kinetics would most likely favor $\mathrm{CH}_{3} \mathrm{O}$ over $\mathrm{CH}_{2} \mathrm{OH}$, since the former deprotonation can be aided by hydrogen-bonded water. With this said, returning to thermodynamics, we find that the conclusion reported by Ferrin and Mavrikakis, ${ }^{9}$ that is, the formation of $\mathrm{CH}_{2} \mathrm{OH}$ is the potential-limiting step, is within the margin of error when including solvent effects.

Knowing that $\mathrm{CH}_{3} \mathrm{O}$ at least does not surpass $\mathrm{CH}_{2} \mathrm{OH}$ in energy significantly for the investigated solvent models, we assume that the rest of the reaction mechanism continues from $\mathrm{CH}_{2} \mathrm{OH}$ (the next step is $\mathrm{CH}_{2} \mathrm{O}$ - formaldehyde-in either case) as in eq 2. Figure 3 shows the change in step limiting potential of the different reaction intermediates as the number of explicit water increases in the presence of an implicit solvent model (Figure 3a) and without (Figure 3b). For some of the intermediates with implicit solvent $\left(\mathrm{CH}_{2} \mathrm{OH}, \mathrm{CH}_{2} \mathrm{O}, \mathrm{CHO}\right.$, and $\mathrm{CHOO}$ ), the dependence on explicit water molecules is small, while $\mathrm{HCOOH}$ converges somewhat with six $\mathrm{H}_{2} \mathrm{O}$ and $\mathrm{CO}_{2}$ seems to require more. The former group has in common that the general water coordination is similar before and after the deprotonation, while $\mathrm{HCOOH}$ binds to more water and $\mathrm{CO}_{2}$ to fewer compared to the previous reaction step. When comparing the results without implicit solvent in Figure $3 \mathrm{~b}$, similar general behavior can be seen while the spread is greater and convergence with respect to water molecules is further away. On the basis of vacuum calculations, it is not clear whether the formation of $\mathrm{CH}_{2} \mathrm{OH}$ or $\mathrm{CHOO}$ is the potentialdetermining step even after six water molecules. In contrast, $\mathrm{CH}_{2} \mathrm{OH}$ formation is always potential-determining when including implicit solvent. The outlier for $\mathrm{CH}_{2} \mathrm{OH}$ with four $\mathrm{H}_{2} \mathrm{O}$ is caused by the outlier in the discussion of Figure $2 \mathrm{a}$.

As the number of explicit water molecules increases, the use of the implicit solvent model for bulk water should represent a more accurate description of the full system. In Figure 4, we 
compare the reaction landscape of MOR with six explicit water molecules and the use of the implicit solvent model with the most simple model, that is, no explicit water and no implicit solvent, which is very often used in the literature. It is clear that the simple model qualitatively captures the reaction landscape. For instance, the potential-determining step (PDS), i.e., the step with the largest equilibrium potential, is the same in the two models, i.e., the first deprotonation step. In the simple model, the PDS has a value of $0.55 \mathrm{eV}$, while in the more realistic model, the PDS equals a value of $0.59 \mathrm{eV}$. What the simple model misses is that $\mathrm{CH}_{3} \mathrm{O}$ is more likely to be formed.

Cyclic voltammetry was performed to investigate the onset potential and activity of methanol oxidation, shown in Figure 5. The corresponding $\mathrm{pH}$ for the $\mathrm{KOH}$ and $\mathrm{HClO}_{4}$ solution is

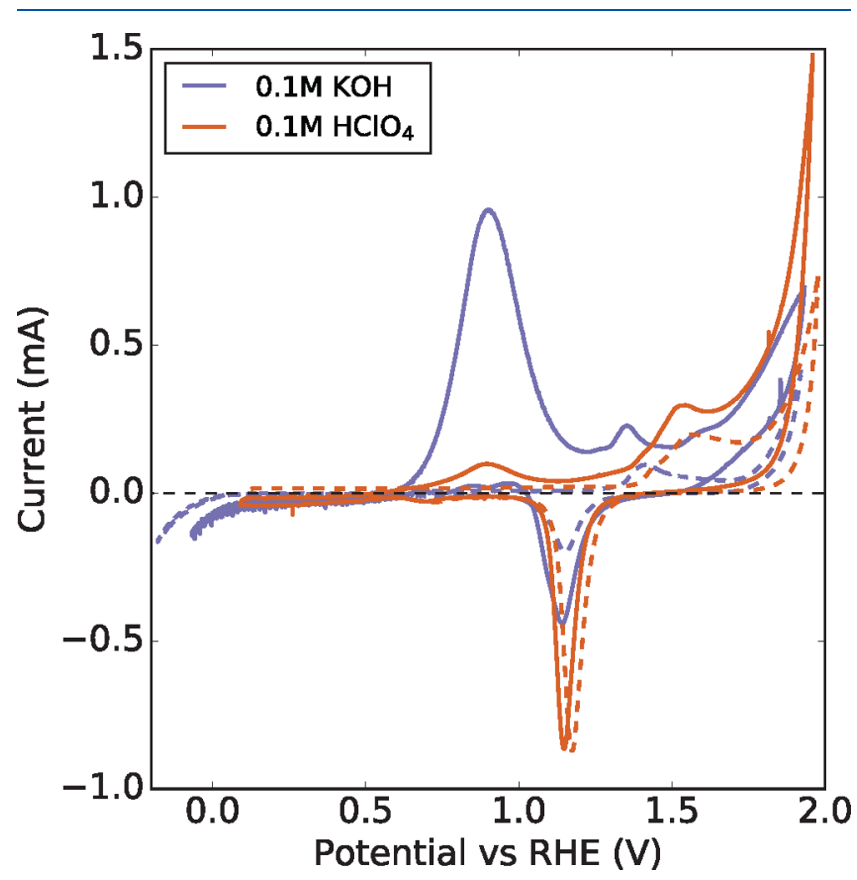

Figure 5. Cyclic voltammogram of methanol with a polycrystalline gold electrode in $0.1 \mathrm{M} \mathrm{KOH}$ (blue) and $0.1 \mathrm{M} \mathrm{HClO}_{4}$ (red). The dashed curves indicate blank solutions. Scan rate: $100 \mathrm{mV} / \mathrm{s}$.

13 and 1 , respectively. We find that the activity of methanol electrooxidation depends heavily on $\mathrm{pH}$, which is similar to our previous results with glycerol. ${ }^{36}$ The main reason for low activity in acid is competitive adsorption from the negative anions since the noncharged methanol adsorption on gold is weak. In alkaline media, however, alcohols are at least partially protolyzed in solution, which facilitate adsorption. ${ }^{37}$ As it can be seen in Figure 5, there is a large oxidation peak on the anodic scan in $\mathrm{KOH}$ at $1.0 \mathrm{~V}$ vs RHE, while the corresponding peak in $\mathrm{HClO}_{4}$ is much smaller. The onset potential in both acidic and alkaline solution is around $0.6 \mathrm{~V}$ vs RHE. The onset potential should be higher than the theoretical limiting potential due to kinetics, so we find a reasonable agreement with the theoretical results. Unfortunately, because the two different PDS values are so close in energy, the experimental onset potential cannot determine which model is correct.

\section{CONCLUSIONS}

While for several intermediates, implicit solvation gives limiting potentials with a small dependence on the number of explicit water molecules, $\mathrm{CH}_{3} \mathrm{O}, \mathrm{HCOOH}$, and $\mathrm{CO}_{2}$ are clearly affected. These formations require 3-4, 5-6, and $>6$ explicit water molecules, respectively, to reach convergence. The probable reason for the dependence is the increase $\left(\mathrm{CH}_{3} \mathrm{O}\right.$ and $\mathrm{HCOOH})$ and decrease $\left(\mathrm{CO}_{2}\right)$ of hydrogen bonds to the surrounding water molecules compared to the previous intermediate. Thus, we find that VASPsol should be combined with some explicit water molecules for modeling this kind of system.

Within the limitations of our model, with vacuum singlepoint calculations on implicit-solvent relaxed structures, we find that even explicit solvation with six water molecules is insufficient to model solvent effects on methanol oxidation. This model cannot clearly identify the potential-determining step and only gives some qualitative indications of solvation effects on the reaction landscape. We find thus that there is no benefit of including only explicit solvation.

The use of more realistic solvent models has some effects on the methanol electrooxidation reaction mechanism. When comparing the most simple model, i.e., no explicit water and no implicit solvent, with the most advanced model, i.e., six water molecules and an implicit solvent, the main difference lies in the explicit-solvent dependent steps of $\mathrm{CH}_{3} \mathrm{O}, \mathrm{HCOOH}$, and $\mathrm{CO}_{2}$ formation. As a consequence, the advanced model indicates that $\mathrm{CH}_{3} \mathrm{O}$ is competitive as a potential-determining step, while the simple model rejects it. On the other hand, both models identify the formation of $\mathrm{CH}_{2} \mathrm{OH}$ as the potentialdetermining step with very similar theoretical limiting potentials ( 0.55 and $0.59 \mathrm{~V}$ vs RHE). Both of these agree with our experimental results of onset at $0.6 \mathrm{~V}$, which should be higher due to kinetics.

\section{ASSOCIATED CONTENT}

\section{Supporting Information}

The Supporting Information is available free of charge at https://pubs.acs.org/doi/10.1021/acs.jpcc.0c08923.

Solvent effects on methanol electrooxidation on gold (PDF)

Structure and data files (ZIP)

\section{AUTHOR INFORMATION}

\section{Corresponding Author}

Anders Hellman - Department of Physics and Competence Centre for Catalysis, Chalmers University of Technology, SE41296 Göteborg, Sweden; Phone: +46 3177256 11; Email: anders.hellman@chalmers.se

\section{Authors}

Mikael Valter - Department of Physics, Chalmers University of Technology, SE-41296 Göteborg, Sweden; 이이.org/ 0000-0002-7718-3525

Björn Wickman - Department of Physics and Competence Centre for Catalysis, Chalmers University of Technology, SE-

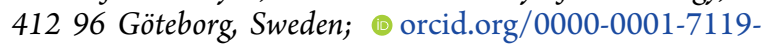
9529

Complete contact information is available at: https://pubs.acs.org/10.1021/acs.jpcc.0c08923

\section{Notes}

The authors declare no competing financial interest. 


\section{ACKNOWLEDGMENTS}

The authors gratefully acknowledge support from Swedish Research Council and Formas. The electronic structure calculations were performed on resources provided by the Swedish National Infrastructure for Computing (SNIC) at NSC and UPPMAX. The authors further thank Adam Arvidsson for making the TOC graphic.

\section{REFERENCES}

(1) Aricò, A. S.; Srinivasan, S.; Antonucci, V. DMFCs: From Fundamental Aspects to Technology Development. Fuel Cells 2001, 1, $133-161$.

(2) Zhao, X.; Yin, M.; Ma, L.; Liang, L.; Liu, C.; Liao, J.; Lu, T.; Xing, W. Recent Advances in Catalysts for Direct Methanol Fuel Cells. Energy Environ. Sci. 2011, 4, 2736-2753.

(3) Kakati, N.; Maiti, J.; Lee, S. H.; Jee, S. H.; Viswanathan, B.; Yoon, Y. S. Anode Catalysts for Direct Methanol Fuel Cells in Acidic Media: Do We Have Any Alternative for Pt or Pt-Ru? Chem. Rev. 2014, 114, 12397-12429.

(4) Joghee, P.; Malik, J. N.; Pylypenko, S.; O’Hayre, R. A Review on Direct Methanol Fuel Cells - In the Perspective of Energy and Sustainability. MRS Energy Sustain. 2015, 2, E3.

(5) Olah, G. A. Beyond Oil and Gas: The Methanol Economy. Angew. Chem., Int. Ed. 2005, 44, 2636-2639.

(6) Iwasita, T. Electrocatalysis of Methanol Oxidation. Electrochim. Acta 2002, 47, 3663-3674.

(7) Sakong, S.; Groß, A. The Importance of the Electrochemical Environment in the Electro-Oxidation of Methanol on $\mathrm{Pt}(111)$. ACS Catal. 2016, 6, 5575-5586.

(8) Ferrin, P.; Nilekar, A. U.; Greeley, J.; Mavrikakis, M.; Rossmeisl, J. Reactivity Descriptors for Direct Methanol Fuel Cell Anode Catalysts. Surf. Sci. 2008, 602, 3424-3431.

(9) Ferrin, P.; Mavrikakis, M. Structure Sensitivity of Methanol Electrooxidation on Transition Metals. J. Am. Chem. Soc. 2009, 131, 14381-14389.

(10) Rossmeisl, J.; Ferrin, P.; Tritsaris, G. A.; Nilekar, A. U.; Koh, S.; Bae, S. E.; Brankovic, S. R.; Strasser, P.; Mavrikakis, M. Bifunctional Anode Catalysts for Direct Methanol Fuel Cells. Energy Environ. Sci. 2012, 5, 8335-8342.

(11) Tritsaris, G. A.; Rossmeisl, J. Methanol Oxidation on Model Elemental and Bimetallic Transition Metal Surfaces. J. Phys. Chem. C 2012, 116, 11980-11986.

(12) Garcia-Ratés, M.; García-Muelas, R.; López, N. Solvation Effects on Methanol Decomposition on $\mathrm{Pd}(111), \mathrm{Pt}(111)$, and $\mathrm{Ru}(0001)$. J. Phys. Chem. C 2017, 121, 13803-13809.

(13) Gao, W.; Keith, J. A.; Anton, J.; Jacob, T. Theoretical Elucidation of the Competitive Electro-oxidation Mechanisms of Formic Acid on Pt(111). J. Am. Chem. Soc. 2010, 132, 18377-18385.

(14) Gao, W.; Mueller, J. E.; Jiang, Q.; Jacob, T. The Role of CoAdsorbed $\mathrm{CO}$ and $\mathrm{OH}$ in the Electrooxidation of Formic Acid on $\mathrm{Pt}(111)$. Angew. Chem., Int. Ed. 2012, 51, 9448-9452.

(15) Gao, W.; Song, E. H.; Jiang, Q.; Jacob, T. Revealing the Active Intermediates in the Oxidation of Formic Acid on $\mathrm{Au}$ and $\mathrm{Pt}(111)$. Chem. - Eur. J. 2014, 20, 11005-11012.

(16) Heenen, H. H.; Gauthier, J. A.; Kristoffersen, H. H.; Ludwig, T.; Chan, K. Solvation at Metal/water Interfaces: An Ab Initio Molecular Dynamics Benchmark of Common Computational Approaches. J. Chem. Phys. 2020, 152, 144703.

(17) Calle-Vallejo, F.; de Morais, R. F.; Illas, F.; Loffreda, D.; Sautet, P. Affordable Estimation of Solvation Contributions to the Adsorption Energies of Oxygenates on Metal Nanoparticles. J. Phys. Chem. C 2019, 123, 5578-5582.

(18) Ludwig, T.; Gauthier, J. A.; Brown, K. S.; Ringe, S.; Nørskov, J. K.; Chan, K. Solvent-Adsorbate Interactions and Adsorbate-Specific Solvent Structure in Carbon Dioxide Reduction on a Stepped $\mathrm{Cu}$ Surface. J. Phys. Chem. C 2019, 123, 5999-6009.

(19) Schweitzer, B.; Steinmann, S. N.; Michel, C. Can Microsolvation Effects Be Estimated from Vacuum Computations? A Case-
Study of Alcohol Decomposition at the $\mathrm{H}_{2} \mathrm{O} / \mathrm{Pt}(111)$ Interface. Phys. Chem. Chem. Phys. 2019, 21, 5368-5377.

(20) Kresse, G.; Hafner, J. Ab Initio Molecular Dynamics for Liquid Metals. Phys. Rev. B: Condens. Matter Mater. Phys. 1993, 47, 558-561.

(21) Kresse, G.; Furthmüller, J. Efficiency of Ab-Initio Total Energy Calculations for Metals and Semiconductors Using a Plane-Wave Basis Set. Comput. Mater. Sci. 1996, 6, 15-50.

(22) Kresse, G.; Furthmüller, J. Efficient Iterative Schemes for Ab Initio Total-Energy Calculations Using a Plane-Wave Basis Set. Phys. Rev. B: Condens. Matter Mater. Phys. 1996, 54, 11169-11186.

(23) KlimeŠ, J.; Bowler, D. R.; Michaelides, A. Chemical Accuracy for the van Der Waals Density Functional. J. Phys.: Condens. Matter 2010, 22, 022201.

(24) KlimeŠ, J.; Bowler, D. R.; Michaelides, A. Van Der Waals Density Functionals Applied to Solids. Phys. Rev. B: Condens. Matter Mater. Phys. 2011, 83, 195131.

(25) Becke, A. D. On the Large-Gradient Behavior of the Density Functional Exchange Energy. J. Chem. Phys. 1986, 85, 7184-7187.

(26) Baltrusaitis, J.; Valter, M.; Hellman, A. Geometry and Electronic Properties of Glycerol Adsorbed on Bare and TransitionMetal Surface-Alloyed Au(111): A Density Functional Theory Study. J. Phys. Chem. C 2016, 120, 1749-1757.

(27) Blöchl, P. E. Projector Augmented-Wave Method. Phys. Rev. B: Condens. Matter Mater. Phys. 1994, 50, 17953-17979.

(28) Mathew, K.; Kolluru, V. S. C.; Hennig, R. G. VASPsol: Implicit Solvation and Electrolyte Model for Density-Functional Theory. https://github.com/henniggroup/VASPsol, 2018.

(29) Mathew, K.; Sundararaman, R.; Letchworth-Weaver, K.; Arias, T. A.; Hennig, R. G. Implicit Solvation Model for Density-Functional Study of Nanocrystal Surfaces and Reaction Pathways. J. Chem. Phys. 2014, 140, 084106.

(30) Monkhorst, H. J.; Pack, J. D. Special Points for Brillouin-Zone Integrations. Phys. Rev. B 1976, 13, 5188-5192.

(31) Nørskov, J. K.; Rossmeisl, J.; Logadottir, A.; Lindqvist, L.; Kitchin, J. R.; Bligaard, T.; Jónsson, H. Origin of the Overpotential for Oxygen Reduction at a Fuel-Cell Cathode. J. Phys. Chem. B 2004, 108, 17886-17892.

(32) Chan, K.; Nørskov, J. K. Electrochemical Barriers Made Simple. J. Phys. Chem. Lett. 2015, 6, 2663-2668.

(33) Skúlason, E.; Karlberg, G. S.; Rossmeisl, J.; Bligaard, T.; Greeley, J.; Jónsson, H.; Nørskov, J. K. Density Functional Theory calculations for the Hydrogen Evolution Reaction in an Electrochemical Double Layer on the Pt (111) Electrode. Phys. Chem. Chem. Phys. 2007, 9, 3241-3250.

(34) Sundararaman, R.; Goddard, W. A., III; Arias, T. A. Grand Canonical Electronic Density-Functional Theory: Algorithms and Applications to Electrochemistry. J. Chem. Phys. 2017, 146, 114104.

(35) Akhade, S. A.; Bernstein, N. J.; Esopi, M. R.; Regula, M. J.; Janik, M. J. A Simple Method to Approximate Electrode PotentialDependent Activation Energies Using Density Functional Theory. Catal. Today 2017, 288, 63-73.

(36) Valter, M.; Busch, M.; Wickman, B.; Gronbeck, H.; Baltrusaitis, J.; Hellman, A. Electrooxidation of Glycerol on Gold in Acidic Medium: a Combined Experimental and DFT Study. J. Phys. Chem. C 2018, 122, 10489-10494.

(37) Kwon, Y.; Lai, S. C. S.; Rodriguez, P.; Koper, M. T. M. Electrocatalytic Oxidation of Alcohols on Gold in Alkaline Media: Base or Gold Catalysis? J. Am. Chem. Soc. 2011, 133, 6914-6917. 